\title{
ROBUST CONTROL OF SMART STRUCTURES WITH OPTIMALLY PLACED ACTUATORS AND SENSORS
}

\author{
Amalia G. Moutsopoulou ${ }^{1}$, Georgia Foutsitzi ${ }^{2}$, Christos Gogos ${ }^{3}$ and Georgios E. \\ Stavroulakis $^{4}$ \\ ${ }^{1}$ Department of Civil Engineering, Technological Educational Institute of Crete, \\ Estavromenos Heraklion Crete, Greece \\ e-mail: amalia@staff.teicrete.gr \\ ${ }^{2,3}$ Department of Accounting and Finance, Technological Educational \\ Institution of Epirus, TEI Campus-Psathaki, GR-48100 Preveza, Greece \\ e-mails: gfoutsi@teiep.gr, cgogos@teiep.gr \\ ${ }^{4}$ Department of Production Engineering and Management, \\ Technical University of Crete, GR-73100 Chania, Greece \\ e-mail:gestavr@dpem.tuc.gr
}

Keywords: Robust Control, Vibration Control, Piezoelectric beam, Actuator Placement

\begin{abstract}
.
The objective of this work is to design robust controller for smart structures to control its response under the influence of external excitation. An accurate model for the analysis of smart composite beams with surface bonded piezoelectric sensors and actuators patches is considered. Optimal placement of five piezoelectric sensor/actuator pairs are found, by exhaustively examining all possible configurations in order to suppress the first six modes of vibration. The LQR performance index is used as objective function to locate the sensor/actuator pairs. Vibration reduction for the cantilever beam with piezoelectric patches bonded in the optimal location was investigated to attenuate the first six modes of vibration using a $H_{\text {infinity }}$ scheme. After modeling multiplicative uncertainty, the augmented uncertain plant is obtained; an op-

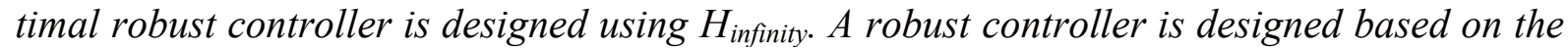
augmented plant composed of the nominal model and it's accompanied uncertain. Robust and nominal performances of designed controllers are achieved for perturbed plants and results were compared.
\end{abstract}




\section{INTRODUCTION}

The trend of civil engineering design requires structures to become lighter, more flexible and stronger, so in recent years, the light structures have been widely used in various engineering applications [1]. The use of active control techniques for the suppression of vibrations of very light structures is a very important target in many applications, where the additional masses of stiffeners or dampers should be avoided. Active techniques are also more suitable in cases where the disturbance to be cancelled or the properties of the controlled system vary with time [2]. In practice, any structure that deforms under some loading can be regarded as flexible structure and is a distributed parameter system. This implies that vibration at one point is related to vibration at the rest of the points over the structure [3]. Thus, it is desirable to use appropriate sensors and actuators. Piezoelectric sensors and actuators are extensively employed in many practical applications such as smart structures due to their lightness and their capability of coupling strain and electric fields. In order to control structural vibrations, piezoelectric sensors and actuators can be easily bonded on the vibrating structure [4]. Vibration suppression of a flexible structure can be optimized by locating piezoelectric sensors and actuators in efficient locations.

This work is concerned with active vibration reduction of a beam, mounted rigidly along one edge to form a cantilever. Optimal placement of five piezoelectric sensor/actuator pairs to thirty possible locations in order to suppress the first six modes of vibration is obtained by fully examining all possible configurations. The LQR performance index is used as objective function to locate the sensor/actuator pairs. The beam, with piezoelectric sensor/actuator pairs bonded to its surfaces, is modelled using the super-convergent FE approach which includes extension, bending and rotation degrees of freedom [10]. Vibration reduction for the cantilever beam with piezoelectric patches bonded in the optimal location was investigated to attenuate the first six modes of vibration using an $H_{\text {infinity }}$ control scheme.

\section{DYNAMIC MODELING OF STRUCTURE}

For designing a controller, a structure consists of a cantilever beam with five surface bonded piezoelectric pairs is considered. The patches are used as actuators and sensors and they are attached symmetrically to either side of the beam, thus collocating the actuator and sensor. For the analysis of the cantilevered composite structure, a super-convergent finite element (FE) model is used [10].

\subsection{Modelling}

The dynamical description of the system is given by

$$
M \because .+D ;,+K_{u и} \chi(t)=f_{m}(t)+f_{e}(t)
$$

where $M$ is the generalized mass matrix, $D$ is the viscous damping matrix, $K_{u u}$ is the generalized stiffness matrix, $f_{m}$ is the external loading vector and $f_{e}$ is the generalized control force vector produced by electromechanical coupling effects. The independent variable $\chi(t)$ is composed of the axial displacements $u_{i}$, transversal deflections $w_{i}$ and rotations $\psi_{i},[10]$.

Furthermore to express $f_{e}(t)$ as $B u(t)$ we write it as $f_{e}^{*} u$, where $f_{e}^{*}$ is the piezoelectric force for a unit applied on the corresponding actuator, and $u$ represents the voltages on the actuators. Lastly $d(t)=f_{m}(t)$ is the disturbance vector. 
Generally, the number of degrees of freedom of the system in the FE dynamic model is very large. The mode transform can be used to reduce the size of the structural system by using the most significant equation of motions in the new, modal coordinate system. Assume that modal matrix composed of the first $m$ order eigenvectors is $\Phi$, the modal transform can be expressed as

$$
\chi=\Phi q
$$

where $[\Phi]$ is the modal matrix and $q$ is the modal coordinate vector. Substituting the above equation into equations (1) leads to

$$
\bar{M}_{-}^{.}, \quad-{ }_{-}, \quad-\quad(t)=\overline{f_{m}}(t)+\overline{f_{e}}(t)
$$

where $\bar{M}=\Phi^{T} M \Phi, \bar{K}=\Phi^{T} K_{u u} \Phi$ are $m \times m$ diagonal matrices due to the orthogonality of the mode shapes with respect to the mass and stiffness matrices, and $\overline{f_{m}}=\Phi^{T} f_{m}, \overline{f_{e}}=\Phi^{T} f_{e}$. The state space form of the system can be expressed as

where $x$ is the state vector, $A$ is the system matrix, $B$ is the control matrix and $u$ is the input vector.

\subsection{Optimization of Sensors/Actuators Location in Vibration Control}

In active control of smart structures, placement of actuators and sensors (S/A) on the structure is a very important issue in order to achieve most effective actuation. Optimal placement of sensors and actuators over a structure can be different for different criteria. Next, in order to propose performance criteria for S/A locations a criterion based on LQR performance index has been considered.

In this work, it is assumed that every actuator covers exactly the length of one element and possible actuator locations are described through the elements of a vector $\alpha$ of binary variables, whose elements $\alpha_{i}$ are 1 to indicate the presence and 0 the absence of an actuator.

The control vector $u$ can be expressed, using the LQR method, as

$$
u=-R^{-1} B^{T} P,
$$

where $P$ satisfying the following matrix Ricatti equation

$$
A^{T} P+P A-P B R^{-1} B^{T}+Q=0
$$

where $Q$ is a positive semi-definite output weighting matrix and $R$ is a positive input weighting matrix. Since the input matrix $B$ is a function of the locations of the actuators, the system will be changed if the locations of actuators will be changed. in this work, the objective function (criterion) which have been used for the locations of the sensors/actuators pairs is given by [11]

$$
J_{\text {opt }}(a)=\operatorname{trace}[P(a)]
$$

where $\alpha$ represents the location of the actuator-sensor pairs.

Since in this problem setting, there are thirty possible locations and the positions of only five actuators have to be decided, a full enumeration scheme that examined all possible 142506 
combinations was used. In case that the number of actuators or locations gets bigger, then other techniques that handle better the combinatorial explosion problem like Genetic Algorithms and Local Search can be used instead.

\section{STATEMENT OF THE ROBUST CONTROL PROBLEM}

The optimal control problem is initially studied for the nominal system, i.e., the beam with known elastic and piezoelectric properties. A more realistic question concerning the robustness of the control in the presence of defects is also addressed. The fact that the system is influenced by disturbances, such as the wind power, as well as the noise of measurements, is taken into account. The mathematical pattern being used in the designing is an approximation of the real one. Further, a robust control law for the composite beam are designed in order to suppress the vibrations. The response of the controlled nominal and damaged beams is investigated. In order to take into account the incompleteness of the information about the eventual damages and external additional influences a robust $H_{\text {infinity }}$ controller is designed. A system analysis is made on condition that the system is not accurate but includes uncertainty that may be related to some kind of damage $[7,8]$.

For practical applications the algorithm requires several trial-and-error design iterations in order to provide appropriate control voltages, since the piezoelectric actuators can be depolled by high oscillating voltages. The effectiveness of the proposed control strategies is investigated with the help of numerical simulations.

\section{CONTROL DESIGN}

We solve a regulator problem for the smart beam with piezoelectric patches. The objective in this section is to determine the optimal vector of active control forces $u(t)$ subjected to performance criteria and satisfying the dynamical equations of the system, such that to reduce in an optimal way the external excitations. We consider the steady state (infinite time) case, i.e. the optimization horizon is allowed to extend to infinity. We seek a linear state feedback [9]

$$
u=-K x, \quad \text { with constant gain } K \text {. }
$$

The control problem is to keep the beam in equilibrium which means zero displacements and rotations in the face of external disturbances, noise and model inaccuracies, using the available measurements (displacements and rotations) and controls [14, 15].

\subsection{Robustness analysis}

The following three steps are taken in the robustness analysis:

1. Expression of uncertainty set by a mathematical model.

2. Robust stability (RS): check if the system remains stable for all plants within the uncertainty set.

3. Robust performance (RP): if system is robustly stable, check whether performance specifications are met for all plants within the uncertainty set. $[15,16]$

To perform the robustness analysis, the interconnection of Figure 1a,b will be used. 


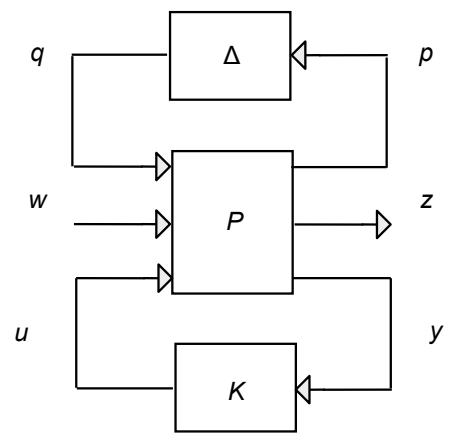

Figure 1a Uncertainty modeling

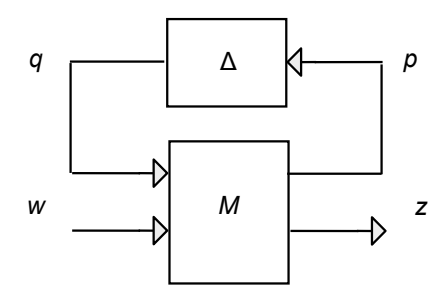

Figure 1b Uncertainty modeling

Here $P$ is the nominal plant defined by figure 1 which includes the uncertainty modelling and $K$ is the calculated $H_{\infty}$ controller. The uncertainty included in $\Delta$ satisfies $\|\Delta\|_{\infty} \leq 1$. Since $K$ is known, figure 1a can be simplified to figure $1 \mathrm{~b}$. $[6,13]$

Given this structure it is known that,

I. The $\operatorname{system}(M, \Delta)$ is robustly stable if,

$$
\sup _{\omega \hat{I} n} \mu_{\Delta}\left(\mathrm{M}_{11}(j \omega)\right)<1
$$

where $\frac{1}{\mu_{\mathrm{B}}(M)}=\left\{\inf _{\Delta \hat{\mathrm{B}}_{\Delta}, \operatorname{det}(I-M \Delta)=0} \bar{\sigma}(\Delta)\right.$ is the structured singular value of $M$ given the structured uncertainty set $\mathrm{B}_{\Delta}$.

II. The system $(M, \Delta)$ exhibits robust performance if,

$$
\sup _{\omega I n} \mu_{\Delta_{a}}(\mathrm{M}(j \omega))<1, \text { where } \Delta_{a}=\left[\begin{array}{cc}
\Delta_{p} & 0 \\
0 & \Delta
\end{array}\right]
$$

and $\Delta_{p}$ has the same structure as $\Delta$ but dimensions corresponding to $(w, z)$.

Unfortunately, only bounds on $\mu$ can be estimated. To proceed let us assume uncertainty in the $M, D$ and $K_{u u}$ matrices of the form,

$$
\begin{gathered}
\bar{M}=M_{0}\left(\mathrm{I}+m_{p} \delta_{M}\right) D=D_{0}\left(\mathrm{I}+d_{p} \delta_{D}\right) \\
\bar{K}_{u u}=K_{0}\left(\mathrm{I}+k_{p} \delta_{K}\right)
\end{gathered}
$$

with

$$
\|\Delta\|_{\infty} \stackrel{\operatorname{def}}{=}\left\|\left[\begin{array}{lll}
\delta_{\mathrm{M}} & & \\
& \delta_{D} & \\
& & \delta_{K}
\end{array}\right]\right\|_{¥}<1
$$

This means we are allowing a percentage deviation from the nominal values. With these definitions Equation (1) becomes,

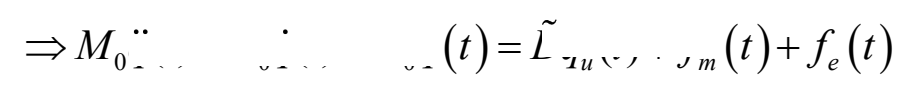


where,

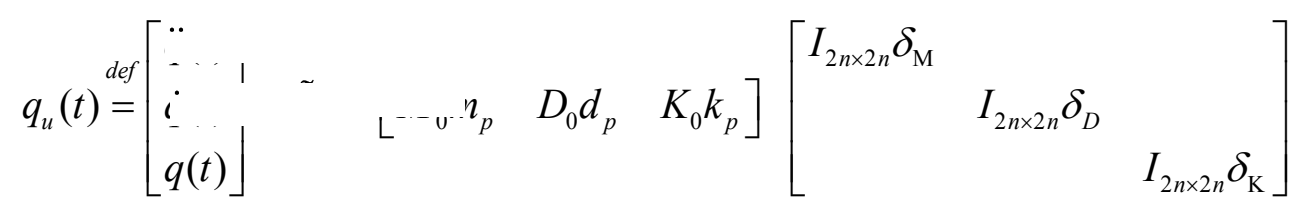

Writing Equation (3) in state space form, gives,

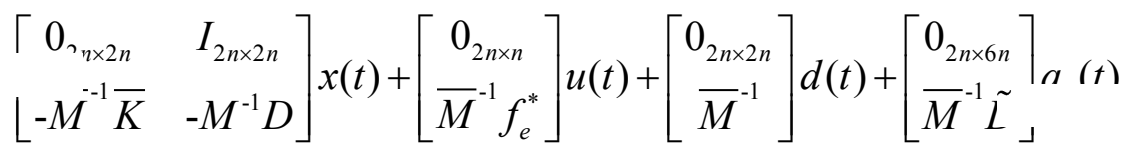

$$
\begin{aligned}
& =\mathrm{A} x(t)+\mathrm{B} u(t)+G d(t)+G_{u} q_{u}(t)
\end{aligned}
$$

\section{RESULTS}

For the numerical simulations a composite beam with piezoelectric layers bonded on its top and bottom is used. The cantilever beam is of dimension $300 \times 25 \times 0.5 \mathrm{~mm}$. The host beam is made of aluminum and the piezoceramic is PZT. The material constants for the aluminum and the piezoceramic are: $Q_{11}=60.0 \mathrm{GPa}, Q_{55}=40.0 \mathrm{GPa}$ and $Q_{11}{ }^{P}=62.0 \mathrm{GPa}, Q_{55}{ }^{\mathrm{P}}=30.0 \mathrm{GPa}$. The piezoelectric constant is $d_{13}=247 \times 10^{-12}$. The thickness of each PZT is $h_{p}=0.35 \mathrm{~mm}$. The length of one piezoelectric patch is assumed to be equal to the length of one finite element. For the finite element analysis the beam is divided into 30 elements and the total number of piezo pairs is assumed to be five. Structural damping is not included in the formulation so that the effectiveness of only the active control in the vibration response can be assessed.

The beam model with the optimal locations of the five sensor-actuator pairs is shown in Fig.2.

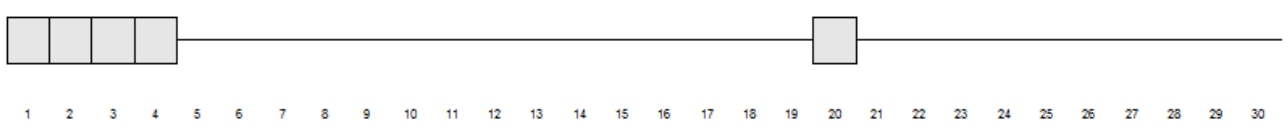

Figure 2. Optimal locations of five sensor-actuator pairs based on the LQR norm.

Next, our aim is to study the response of the composite beam in the presence of defects and damages.

Two kinds of loading are used as disturbances:

- Transient force $4 N$ distributed in the free end of the plate.

- Periodic sinusoidal loading pressure acting on every node on one side of the structure simulating a strong wind.

Let us first investigate the response of the free and LQR-controlled laminated plate with piezoelectric and viscous layers for various parameters of the glue layer. A vertical impulsive 
load is applied at the free-end of the beam. Figures 2a,b show the response of the beam's free end to a constant external force of $4 \mathrm{~N}$ applied to the free end.

The control effort is shown in Figure 2c, where it is seen that both controllers use comparable voltage and are well within the $500 \mathrm{~V}$ limit of piezoelectric actuators. With finer tuning (perhaps by adding a $\mathrm{D}$ term also), the speed of response and other transient characteristics (overshoot) can of course be improved control strategy. The nominal performance is depicted in Figure 3, which shows the response of the uncontrolled and controlled structure using $H_{\infty}$
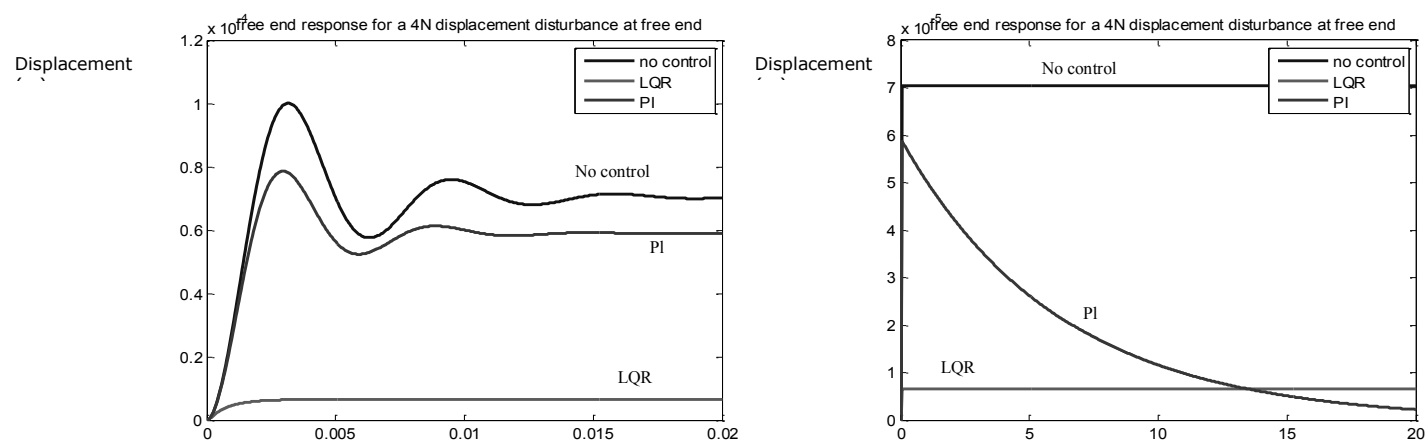

Figure 2a.b. Response of the free vibrating beam with and without control

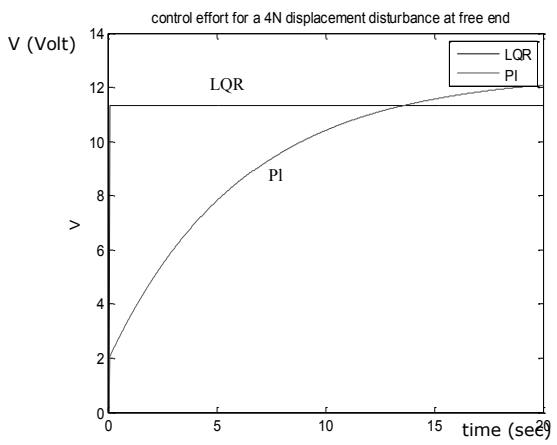

Figure 2c. Control Voltages
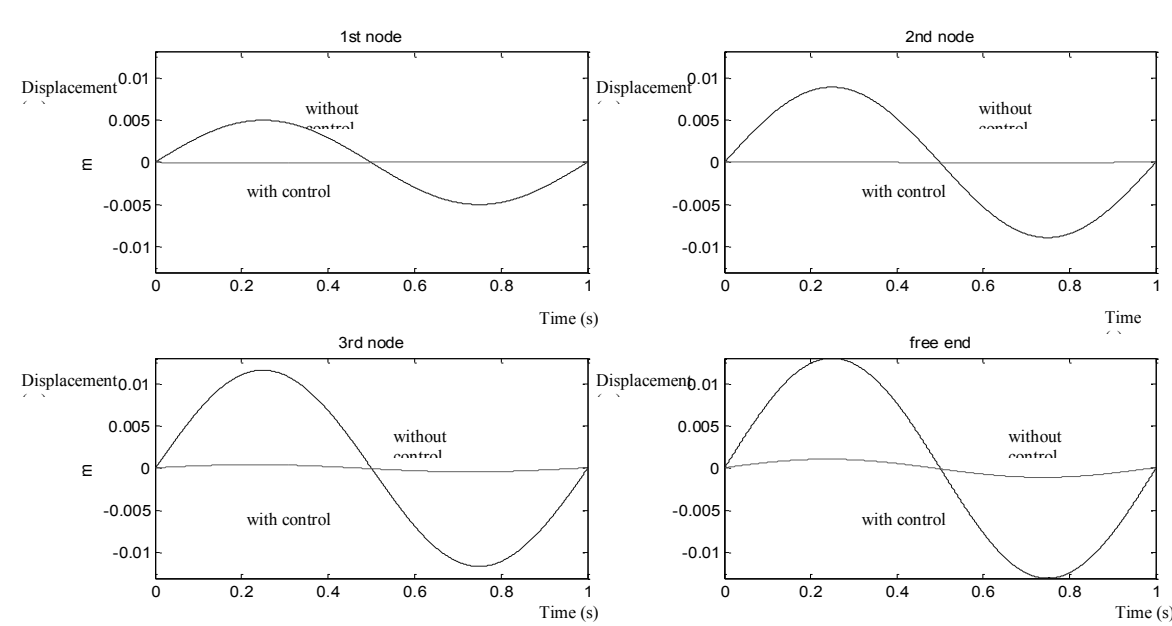

Figure 3 Response of the four nodes vibrating beam with and without control 
The $H_{\infty}$ controller found is of order 36 . The fact that controller order, which is equal to the order of the system, is relatively higher than the order of classical controllers such as LQR has led a number of researchers to develop order reduction algorithms. The most widely used such algorithm, known as HIFOO, has been implemented in a Matlab environment, and is the one used in the following procedure $[6,14,15]$. The general problem is to compute a controller of reduced order $\mathrm{n}<36$ while retaining the performance of the $H_{\infty}$ criterion as well as the behaviour of a full order controller for the given system $[6,14]$. Nonsmooth variation analysis and related computational methods are powerful tools that can be effectively applied to identify local minimizers of the nonconvex optimization problems arising in fixed-order controller design.

The results are shown in Figure 4, where we can see the displacement of the free end of the structure for the open and the closed loop using the $H_{\infty}$ criterion and nonconvex optimization. The results are very satisfactory; we reduced vibrations with a lower order controller. In Figure 5, we take the maximum value of the stress of the structure in the frequency domain.

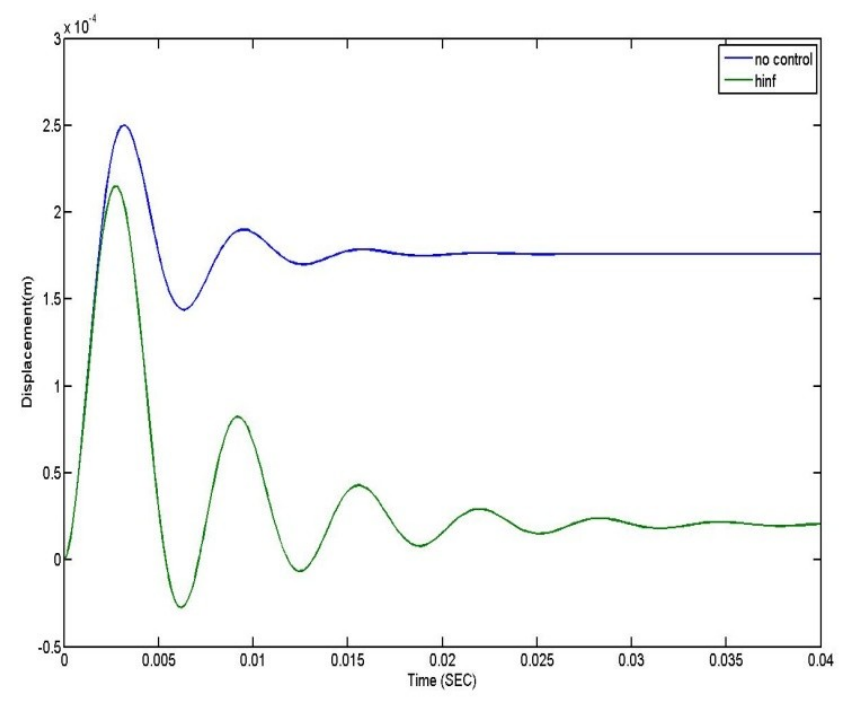

Figure 4 The displacement of the free end of the structure

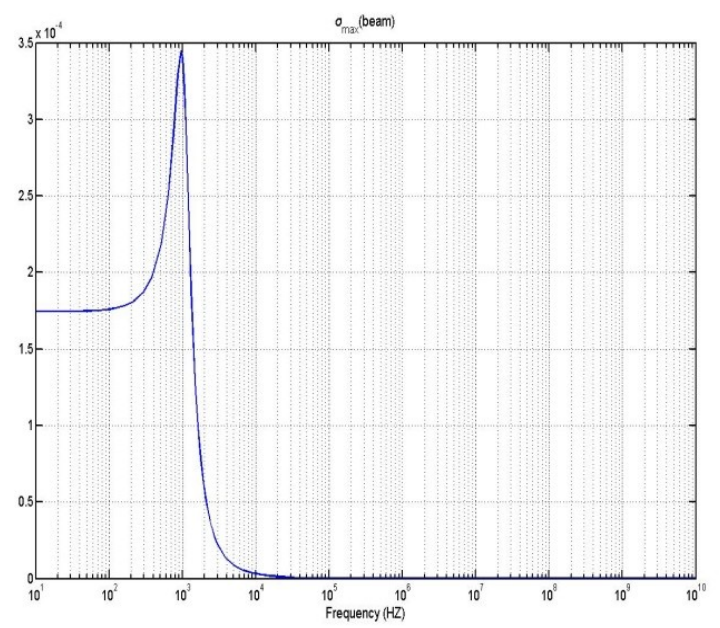

Figure 5 The $\sigma_{\max }$ value for all frequencies 


\section{CONCLUSIONS}

Vibration control of a smart structure with collocated piezoelectric actuator and sensor as a general smart structure has been achieved using optimally placed piezoelectric S/A pairs. The design of the piezoelectric active control using $H_{\text {infinity }}$ control theory for the nominal and damaged laminated plate has been studied. The results show that the proposed model and method are effective and the control behaviour of the beam achieves the predicted characteristics. After the analysis of the system we check the robust stability and system performance. The introduction of uncertainty permits us to keep the structure in service up to given limits of uncertainty.

Acknowledgements: This research has been co-financed by the European Union (European Social Fund - ESF) and Greek national funds through the Operational Program "Education and Lifelong Learning" of the National Strategic Reference Framework (NSRF) - Research Funding Program: ARCHIMEDES III. Investing in knowledge society through the European Social Fund. The authors gratefully acknowledge this support.

\section{REFERENCES}

[1] Y.K. Lee, D. Halim, L. Chen and B. Cazzolato, "Design and Implementation of Spatial Feedback Control on a Flexible Plate", Proceedings of Acoustics, 2004.

[2] Zh.Q. Gu, K.G. Ma, W.D. Chen, “Active vibration control”, Beijing: National Defense Industry Press, 1997.

[3] G. Song, V. Sethi, H.N. Li, "Vibration control of civil structures using piezoceramic smart materials: a review", Eng. Struct., vol.28, pp.1513-1524, 2006.

[4] S. Cen, A.K. Soh, Y.Q. Long, et al., "A new 4-node quadrilateral FE model with variable electrical degrees of freedom for the analysis of piezoelectric laminated composite plates", Compos. Struct, vol.58, pp.583-599, 2002.

[5] B. Bandyopadhyay, T.C. Manjunath, M. Unapathy, Modeling, Control, and Implementation of Smart Structures, Springer, 2007.

[6] J.V. Burke, D. Henron, A.S. Kewis, M.L. Overton, Stabilization via Nonsmooth, Nonconvex Optimization. Automatic Control IEE. 2006, Vol. 5, Issue 11, pp. 17601769.

[7] S.M. Yang, Y.J. Lee, Optimization of non collocated sensor, actuator location and feedback gain and control systems. Smart Materials and Structures J. 1993; 8, 96-102.

[8] B. Miara, G.E. Stavroulakis, V. Valente (eds), Topics on mathematics for smart systems. Proceedings of the European Conference. Rome, Italy, 26-28 October 2006, World Scientific Publishers, Singapore, International, 2007.

[9] A. Moutsopoulou, A. Pouliezos, G.E. Stavroulakis, Modelling with Uncertainty and Robust Control of Smart Beams. Paper 35, Proceedings of the Ninth International Conference on Computational Structures Technology. B.H.V. Topping and M. Papadrakakis, (Editors), Civil Comp Press, 2008.

[10] G. Foutsitzi, E. Hadjigeorgiou, C. Gogos, G.E. Stavroulakis, "Modal shape control of smart composite beams using piezoelectric actuators," in 10th HSTAM (Hellenic Society 
for Theoretical and Applied Mechanics) 2013 International Congress on Mechanics, D. Beskos, G.E. Stavroulakis (Editors), Technical University of Crete Publishing House, Chania 2013.

[11] K.R. Kumar, S. Narayanan, "Active vibration control of beams with optimal placement of piezoelectric sensor/actuator pairs", Smart Mater. Struct. 17055008 (2008)

[12] G.E. Stavroulakis, G. Foutsitzi, E. Hadjigeorgiou, D. Marinova, C.C. Baniotopoulos, Design and robust optimal control of smart beams with application on vibrations suppression Advances in Engineering Software, Volume 36, Issues 11-12, Pages 806-813, November-December 2005.

[13] J.V. Burke, D. Henrion, M.L. Lewis, Overton.HIFOO-a MATLABpackage for fixedordercontroller design and Hinf. optimization. Proceedings of the IFAC Symbosium on Robust Control Design, Toulouse, France, 2006. www.cs.nyu.edu/overton/software/hifoo

[14] O. Bosgra, H. Kwakernaak, Design methods for control systems, Course notes, Dutch Institute for Systems and Control, p.69, 2001.

[15] M. Hou, P.C. Muller, Design of observers for linear systems with unknown inputs, IEEE Trans. on Automatic Control, 37: 871-875, 1992.

[16] D. Marinova, G.E. Stavroulakis, G. Foutsitzi, E. Hadjigeorgiou, E.C. Zacharenakis, Robust design of smart structures taking into account structural defects, Summer School Conference Advanced Problems in Mechanics Russian Academy of Sciences, Editor: D.A. Indeitsev, 288-292, 2004. 